lesions of systemic lupus erythematosus and supportive of an immunopathogenic mechanism for dyslexia.

\title{
LANGUAGE DISORDERS
}

OUTOCME OF AOQUIRED APHASIA

The effects of age at onset, etiology, severity, and type of aphasia on the course and outcome were investigated in a group of 28 aphasic children at the Department of Neurology, University Hospital Rotterdam-Dijkzigt, the Netherlands. Head injury was the cause in eight patients with onset between 4 and 11 years, vascular diseases accounted for seven cases with age of onset between 3 and 13 years, infectious diseases were present in 5 patients with onset between 4 and 12 years, Landau-Kleffner syndrome occurred in six cases with onset at 4-6 years, and cerebral tumor was present in four patients 9-13 years of age. There was no difference in recovery for those children aged above or below 11 years. The very young children with Landau-Kleffner syndrame had a bad prognosis. Six of the eight children with head injury had a favorable outcome, in contrast to those with vascular or infectious disease. Only one of six children with Landau-Kleffner syndrome recovered completely despite normal CT scans. The severity and bilaterality of the lesions showed no significant relation to prognosis of the aphasia. The severity of the cerebral lesion was assessed using a rating scale for CT scans. Most of the children had not recovered completely one year after onset of the aphasia. Recovery was significantly different according to etiological categories. (Loonen MCB, van Dongen HR. Acquired childhood aphasia. Outcome 1 year after onset. Arch Neurol Dec 1990; 47:1324-1328).

COMMENT. In general the outcome of acquired aphasia in childhood is good after mild head injury and a poor outcome may be expected in aphasia due to infectious disorders or Landau-Kleffner syndrome. Bilateral lesions on CT scan are frequently found in patients with infectious and vascular diseases in which aphasia is associated with a poor outcame.

EEG IN LANDAU-KLEFFNER SYNDROME

The EEG was studied in five children with Landau-Kleffner syndrame at the Service de Neurologie I, Hopital Central, Strasbourg, France, and Department de Neurologie C.H.U. Sart Tilman, Liege, Belgium. Day and nighttime EEG video monitoring was performed before and after each change of therapy. One hundred EEGs of 30 minute to two hour duration were recorded on awake patients. Sleep EEGs were obtained during five spontaneous daytime naps, 15 naps induced by amitriptyline, and 65 complete nights of sleep. Spike-wave duration was measured as a percentage of the total sleep period. The EEG in waking patients showed focal and generalized spike-wave discharges on a normal background rhythm. During sleep, discharges increased and bilateral spike-waves occurred more than $85 \%$ of the sleep period. The abnormal EEG and the impairment of higher cognitive function developed and 